\title{
Massive Star Formation: Radiation Transfer Modeling and Multiwavelength Observation
}

\author{
Yichen Zhang ${ }^{1}$, Jonathan Tan ${ }^{1,2}$, Chris McKee $^{3}$, and \\ James De Buizer ${ }^{4}$ \\ ${ }^{1}$ Department of Astronomy, University of Florida, Gainesville, Florida 32611, USA \\ ${ }^{2}$ Departments of Physics, University of Florida, Gainesville, Florida 32611, USA \\ ${ }^{3}$ Departments of Astronomy \& Astrophysics and Physics, University of California, Berkeley, \\ California 94720, USA \\ ${ }^{4}$ SOFIA-USRA, NASA Ames Research Center, MSN211-3, Moffett Field, CA94035, USA \\ email: yczhang@astro.ufl.edu
}

\begin{abstract}
We present a radiation transfer model consistently developed for a core in high pressure environment forming a massive star through core accretion. We compare this model to the massive protostar G35.2-0.74N, including SOFIA observations at 30 and $40 \mu \mathrm{m}$. Good agreement is achieved, showing that a $\sim 30 M_{\odot}$ protostar is forming from a high surface density core via relatively ordered collapse and accretion, driving powerful outflows. This result supports the Core Accretion theory which predicts that massive stars form similarly to low-mass stars.
\end{abstract}

Keywords. stars: formation — ISM: clouds - ISM: jets and outflows

Our radiation transfer (RT) model (Zhang \& Tan 2011; Zhang et al. 2012, ZTM12) is based on the Turbulent Core model of McKee \& Tan (2003): Massive stars form in dense clumps with $\Sigma_{\mathrm{cl}} \sim 1 \mathrm{~g} / \mathrm{cm}^{2}$. In ZTM12, We improved the treatment of the disk by allowing a supply of mass and angular momentum from the infall envelope and their loss to the disk wind. We also developed an approximate disk wind solution. The simulation was performed with the latest version of the Monte Carlo RT code by Whitney et al. (2003, 2012 in prep). Corrections made by adiabatic cooling/heating and advection are included. So are the gas opacities.

We find colors from Spitzer and Herschel data may help to detect and determine the properties of massive protostars. (e.g. at near face-on views, the SED from near-IR to $\sim 70 \mu \mathrm{m}$ is flat. even though dust exists in the outflow cavity.) The inferred bolometric luminosity at a near face-on view can be higher by 1 dex than at near edge-on view (flashlight effect). If the line of sight passes through the outflow cavity and neglecting foreground extinction, the disk can be seen at wavelengths in/longward of the $\mathrm{H}$ band.

In our recent SOFIA-FORCAST observation of massive protostar G35.2-0.74N at 31 and $37 \mu \mathrm{m}$ (Zhang et al. 2012, in prep.), both the near- and far-facing sides of the outflow can be seen. The latter is missing in mid-IR continuum. By fitting both the SED and the axis intensity profiles with our model, the total luminosity is inferred to be $\sim 10^{5}$ after correcting foreground extinction and the flashlight effect. The fitting suggests a $\sim 30 M_{\odot}$ protostar is forming from a high surface density core, $\sim 1 \mathrm{~g} / \mathrm{cm}^{2}$, via ordered collapse and accretion. These indicate a massive star may form similarly to a low-mass star.

\section{References}

McKee, C. F. \& Tan, J. C., 2003, ApJ, 585, 850

Whitney, B. A., Wood, K., Bjorkman, J. E., \& Wolff, M. J., 2003, ApJ, 591, 1049

Zhang, Y. \& Tan, J. C., 2011, ApJ, 733, 55

Zhang, Y., Tan, J. C., \& McKee, C. F., 2012, ApJ, submitted 